\title{
Numerical Solutions of a Class of Second Order Boundary Value Problems on Using Bernoulli Polynomials
}

\author{
Md. Shafiqul Islam ${ }^{1^{*}}$, Afroza Shirin $^{2}$ \\ ${ }^{1}$ Department of Mathematics, Dhaka University, Dhaka, Bangladesh \\ ${ }^{2}$ Department of Mathematics, Bangladesh University of Engineering and Technology, Dhaka, Bangladesh

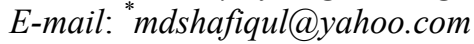 \\ Received January 24, 2011; revised June 3, 2011; accepted June 10, 2011
}

\begin{abstract}
The aim of this paper is to find the numerical solutions of the second order linear and nonlinear differential equations with Dirichlet, Neumann and Robin boundary conditions. We use the Bernoulli polynomials as linear combination to the approximate solutions of 2 nd order boundary value problems. Here the Bernoulli polynomials over the interval $[0,1]$ are chosen as trial functions so that care has been taken to satisfy the corresponding homogeneous form of the Dirichlet boundary conditions in the Galerkin weighted residual method. In addition to that the given differential equation over arbitrary finite domain $[a, b]$ and the boundary conditions are converted into its equivalent form over the interval $[0,1]$. All the formulas are verified by considering numerical examples. The approximate solutions are compared with the exact solutions, and also with the solutions of the existing methods. A reliable good accuracy is obtained in all cases.
\end{abstract}

Keywords: Galerkin Method, Linear and Nonlinear BVP, Bernoulli Polynomials

\section{Introduction}

There are many linear and nonlinear problems in science and engineering, namely second order differential equations with various types of boundary conditions, are solved either analytically or numerically. In the literature of numerical analysis solving a two point second order boundary value problem (BVP) of differential equations, many authors have attempted to obtain higher accuracy rapidly by using a numerous methods. Among various numerical techniques, finite difference method has been widely used but it takes more computational costs to get high accuracy. In this method, a large number of parameters are required and it can not be used to evaluate the value of the desired points between two grid points. For this, Galerkin weighted residual method is widely used to find the approximate results to any point in the domain of the problem.

Since piecewise polynomials can be differentiated and integrated easily, and can be approximated any function to any accuracy desired [1], spline functions have been studied extensively in [2-9]. Solving BVP only with Dirichlet boundary conditions has been attempted in [4] while Bernstein polynomials $[10,11]$ have been used to solve the two point BVP very recently by the authors
Bhatti and Bracken [1] rigorously by the Galerkin method. But it is limited only to second order BVP with Dirichlet boundary conditions, and to first order nonlinear differential equation. On the other hand, Ramadan et al. [2] has studied linear BVP with Neumann boundary conditions using quadratic and cubic polynomial splines, and nonpolynomial splines. We have also found that the linear BVP with Robin (mixed) boundary conditions have been solved using finite difference method [12] and Sinc-Collocation method [13], respectively. Thus except [9], little attention has been given to solve the second order nonlinear BVP with Dirichlet and Neumann as well as Robin boundary conditions. Therefore, the purpose of this paper is to present the Galerkin weighted residual method to solve both linear and nonlinear second order BVP with all types of boundary conditions as well.

Besides spline functions and Bernstein polynomials, there is another type of piecewise continuous polynomials, namely Bernoulli polynomials, has been introduced by Atkinson in [14]. But none has attempted, to the knowledge of the present authors, using these polynomials to solve the second order BVP. Thus we concentrate in this paper rigorously to solve some linear and nonlinear BVP with various types of boundary conditions nu- 
merically, though it is originated in [1].

However, in this paper, we first give an introduction of Bernoulli polynomials, and then we formulate the Galerkin approximation method using Bernoulli polynomials. We derive the individual formulas for each BVP consisting of Dirichlet, Neumann and Robin boundary conditions, respectively. Numerical examples, for both linear and nonlinear boundary value problems, are considered to verify the effectiveness of the derived formulas, and are also compared with the exact solutions. All the computations are performed by MATHEMATICA.

\section{Bernoulli Polynomials}

The Bernoulli polynomials [14, p. 284] of degree $n$ can be defined over the interval $[0,1]$ implicitly by

$$
B r_{n}(x)=\sum_{k=0}^{n}\left(\begin{array}{l}
n \\
k
\end{array}\right) b_{k} x^{n-k}
$$

where, $b_{k}$ are Bernoulli numbers given by

$$
b_{0}=1 \text { and } b_{k}=-\int_{0}^{1} B r_{k}(x) \mathrm{d} x k \geq 1 .
$$

Also Equation (1) can be written explicitly as

$$
\begin{aligned}
B r_{0}(x) & =1 \\
B r_{m}(x) & =\sum_{n=0}^{m} \frac{1}{n+1} \sum_{k=0}^{n}(-1)^{k}\left(\begin{array}{l}
n \\
k
\end{array}\right)(x+k)^{m} \\
& -\sum_{n=0}^{m} \frac{1}{n+1} \sum_{k=0}^{n}(-1)^{k}\left(\begin{array}{l}
n \\
k
\end{array}\right) k^{m}, m \geq 1
\end{aligned}
$$

The first 11 Bernoulli polynomials are given bellow:

$$
\begin{gathered}
B r_{0}(x)=1 \\
B r_{5}(x)=-\frac{x}{6}+\frac{5 x^{3}}{3}-\frac{5 x^{4}}{2}+x^{5} \\
B r_{1}(x)=x \\
B r_{6}(x)=-\frac{x^{2}}{2}+\frac{5 x^{4}}{2}-3 x^{5}+x^{6} \\
B r_{2}(x)=-x+x^{2} \\
B r_{7}(x)=\frac{x}{6}-\frac{7 x^{3}}{6}+\frac{7 x^{5}}{2}-\frac{7 x^{6}}{2}+x^{7} \\
B r_{3}(x)=\frac{x}{2}-\frac{3 x^{2}}{2}+x^{3} \\
B r_{8}(x)=\frac{2 x^{2}}{3}-\frac{7 x^{4}}{3}+\frac{14 x^{6}}{3}-4 x^{7}+x^{8} \\
B r_{4}(x)=x^{2}-2 x^{3}+x^{4}
\end{gathered}
$$

$$
\begin{gathered}
B r_{9}(x)=-\frac{3 x}{10}+2 x^{3}-\frac{21 x^{5}}{5}+6 x^{7}-\frac{9 x^{8}}{2}+x^{9} \\
B r_{10}(x)=-\frac{3 x^{2}}{2}+5 x^{4}-7 x^{6}+\frac{15 x^{8}}{2}-5 x^{9}+x^{10}
\end{gathered}
$$

Since Bernoulli polynomials have special properties at $x=0$ and $x=1: B r_{n}(0)=0, \quad n \geq 1$, and

$B r_{n}(1)=0, n \geq 2$ respectively, so that they can be used as a set of basis functions to satisfy the corresponding homogeneous form of the Dirichlet boundary conditions to derive the matrix formulation of second order BVP over the interval $[0,1]$.

\section{Formulation of Second Order BVP}

We consider the general second order linear BVP [15]:

$$
\begin{gathered}
-\frac{\mathrm{d}}{\mathrm{d} x}\left(p(x) \frac{\mathrm{d} u}{\mathrm{~d} x}\right)+q(x) u=r(x), \quad a<x<b, \\
\alpha_{0} u(a)+\alpha_{1} u^{\prime}(a)=c_{1}, \quad \beta_{0} u(b)+\beta_{1} u^{\prime}(b)=c_{2},
\end{gathered}
$$

where $p(x), q(x)$ and $r(x)$ are specified continuous functions, and $\alpha_{0}, \alpha_{1}, \beta_{0}, \beta_{1}, c_{1}, c_{2}$ are specified numbers. Since our aim is to use the Bernoulli polynomials as trial functions which are derived over the interval $[0,1]$, so the BVP (3) is to be converted to an equivalent problem on $[0,1]$ by replacing $x$ by

$(b-a) x+a$, and thus we have:

$$
\begin{array}{r}
-\frac{\mathrm{d}}{\mathrm{d} x}\left(\tilde{p}(x) \frac{\mathrm{d} u}{\mathrm{~d} x}\right)+\tilde{q}(x) u=\tilde{r}(x), \quad 0<x<1 \\
\alpha_{0} u(0)+\frac{\alpha_{1}}{b-a} u^{\prime}(0)=c_{1}, \quad \beta_{0} u(1)+\frac{\beta_{1}}{b-a} u^{\prime}(1)=c_{2}
\end{array}
$$

where,

$$
\begin{aligned}
& \tilde{p}(x)=\frac{1}{(b-a)^{2}} p((b-a) x+a), \\
& \tilde{q}(x)=q((b-a) x+a), \\
& \tilde{r}(x)=r((b-a) x+a)
\end{aligned}
$$

Using Bernoulli polynomials, $B r_{i}(x)$ in Equation (2), we assume an approximate solution in a form,

$$
\tilde{u}(x)=\sum_{i=0}^{n} a_{i} B r_{i}(x), \quad n \geq 1,
$$

Now the weighted residual equations corresponding to the differential Equation (4a) given by

$$
\begin{aligned}
& \int_{0}^{1}\left[-\frac{\mathrm{d}}{\mathrm{d} x}\left(\tilde{p}(x) \frac{\mathrm{d} \tilde{u}}{\mathrm{~d} x}\right)+\tilde{q}(x) \tilde{u}-\tilde{r}(x)\right] B r_{j}(x) \mathrm{d} x=0, \\
& j=1,2,3, \cdots, n
\end{aligned}
$$

Since from (5), we have 


$$
\begin{aligned}
& \frac{\mathrm{d} \tilde{u}}{\mathrm{~d} x}=\sum_{i=0}^{n} a_{i} \frac{\mathrm{d} B r_{i}}{\mathrm{~d} x} \\
& \tilde{u}(0)=\sum_{i=0}^{n} a_{i} B r_{i}(0) \text { and } \tilde{u}(1)=\sum_{i=0}^{n} a_{i} B r_{i}(1)
\end{aligned}
$$

After minor simplification, from (6) we can obtain

$$
\begin{gathered}
\sum_{i=0}^{n}\left[\int_{0}^{1}\left[\tilde{p}(x) \frac{\mathrm{d} B r_{i}}{\mathrm{~d} x} \frac{\mathrm{d} B r_{j}}{\mathrm{~d} x}+\tilde{q}(x) B r_{i}(x) B r_{j}(x)\right] \mathrm{d} x\right. \\
\left.+\frac{\beta_{0}(b-a) \tilde{p}(1) B r_{i}(1) B r_{j}(1)}{\beta_{1}}\right] a_{i} \\
\left.-\frac{\alpha_{0}(b-a) \tilde{p}(0) B r_{i}(0) B r_{j}(0)}{\alpha_{1}}\right] \int_{0}^{1} \tilde{r}(x) B r_{j}(x) \mathrm{d} x+\frac{c_{2}(b-a) \tilde{p}(1) B r_{j}(1)}{\beta_{1}} \\
-\frac{c_{1}(b-a) \tilde{p}(0) B r_{j}(0)}{\alpha_{1}}
\end{gathered}
$$

or, equivalently in matrix form,

$$
\sum_{i=0}^{n} D_{i, j} a_{i}=F_{j}, j=0,1,2, \cdots, n
$$

where,

$$
\begin{aligned}
D_{i, j}= & \int_{0}^{1}\left[\tilde{p}(x) \frac{\mathrm{d} B r_{i}}{\mathrm{~d} x} \frac{\mathrm{d} B r_{j}}{\mathrm{~d} x}+\tilde{q}(x) B r_{i}(x) B r_{j}(x)\right] \mathrm{d} x \\
& +\frac{\beta_{0}(b-a) \tilde{p}(1) B r_{i}(1) B r_{j}(1)}{\beta_{1}} \\
& -\frac{\alpha_{0}(b-a) \tilde{p}(0) B r_{i}(0) B r_{j}(0)}{\alpha_{1}} \\
i, j= & 0,1,2, \cdots, n \\
F_{j}= & \int_{0}^{1} \tilde{r}(x) B r_{j}(x) \mathrm{d} x+\frac{c_{2}(b-a) \tilde{p}(1) B r_{j}(1)}{\beta_{1}} \\
& -\frac{c_{1}(b-a) \tilde{p}(0) B r_{j}(0)}{\alpha_{1}}, \\
j= & 0,1,2, \cdots, n
\end{aligned}
$$

Solving the system (8a), we find the values of the parameters $a_{i}(i=0,1,2, \cdots, n)$, and then substituting these parameters in (5), we get the approximate solution of the BVP (4). If we replace $x$ by $\frac{x-a}{b-a}$ in $\tilde{u}(x)$, then we get the desired approximate solution of the BVP (3).

The absolute error, $E$ of this formulation is defined by

$$
E=|u(x)-\tilde{u}(x)| .
$$

Now we discuss the various types of BVP using dif- ferent boundary conditions as follows:

Case 1: The matrix formulation with the Robin (mixed) boundary conditions, (i.e., $\alpha_{0} \neq 0, \alpha_{1} \neq 0, \beta_{0} \neq 0$, $\left.\beta_{1} \neq 0\right)$, are already defined in Equations (8).

Case 2: The matrix formulation of the differential equation (3a) with the Dirichlet boundary conditions (i.e., $\left.\alpha_{0} \neq 0, \alpha_{1} \neq 0, \quad \beta_{0} \neq 0, \quad \beta_{1} \neq 0\right)$, is given by

$$
\sum_{i=2}^{n} D_{i, j} \quad a_{i}=F_{j}, j=2,3, \cdots, n
$$

where,

$$
\begin{aligned}
D_{i, j}= & \int_{0}^{1}\left[\tilde{p}(x) \frac{\mathrm{d} B r_{i}}{\mathrm{~d} x} \frac{\mathrm{d} B r_{j}}{\mathrm{~d} x}+\tilde{q}(x) B r_{i}(x) B r_{j}(x)\right] \mathrm{d} x, \\
i, j= & 2,3, \cdots, n . \\
F_{j}= & \int_{0}^{1} \tilde{r}(x) B r_{j}(x) \mathrm{d} x \\
& -\int_{0}^{1}\left[\tilde{p}(x) \frac{\mathrm{d} \theta_{0}}{\mathrm{~d} x} \frac{\mathrm{d} B r_{j}}{\mathrm{~d} x}+\tilde{q}(x) \theta_{0}(x) B r_{j}(x)\right] \mathrm{d} x, \\
j= & 2,3, \cdots, n
\end{aligned}
$$

Case 3: The approximate solution of the differential equation (3a) consisting of Neumann boundary conditions (i.e., $a_{0}=0, a_{1} \neq 0, \beta_{0}=0, \beta_{1} \neq 0$, ) can be obtained by putting $a_{0}=0$ and $\beta_{0}=0$, in the Equation (8) as

$$
\sum_{i=0}^{n} D_{i, j} \quad a_{i}=F_{j}, j=0,1,2, \cdots, n
$$

where

$$
\begin{aligned}
D_{i, j}= & \int_{0}^{1}\left[\tilde{p}(x) \frac{\mathrm{d} B r_{i}}{\mathrm{~d} x} \frac{\mathrm{d} B r_{j}}{\mathrm{~d} x}+\tilde{q}(x) B r_{i}(x) B r_{j}(x)\right] \mathrm{d} x, \\
i, j= & 0,1,2, \cdots, n \\
F_{j} & =\int_{0}^{1} \tilde{r}(x) B r_{j}(x) \mathrm{d} x+\frac{c_{2}(b-a) \tilde{p}(1) B r_{j}(1)}{\beta_{1}} \\
& -\frac{c_{1}(b-a) \tilde{p}(0) B r_{j}(0)}{\alpha_{1}}, \\
j & =0,1,2, \cdots, n
\end{aligned}
$$

Similar formulation for nonlinear BVP using the Bernoulli polynomials can be derived, which will be discussed through numerical examples in the next section.

\section{Numerical Examples}

In this section, we explain four linear and two nonlinear boundary value problems which are available in the existing literatures, considering three types of boundary conditions to verify the effectiveness of the present for- 
mulations described in the previous sections. The convergence of each linear BVP is calculated by

$$
E=\left|u_{n+1}(x)-u_{n}(x)\right|<\delta,
$$

where $u_{n}(x)$ denotes the approximate solution by the proposed method using $n$-th degree polynomial approximation. The convergence of nonlinear BVP is assumed when the absolute error of two consecutive iterations is recorded below the convergence criterion $\delta$ such that

$$
\left|\tilde{u}^{N+1}-\tilde{u}^{N}\right|<\delta
$$

where $N$ is the Newton's iteration number and $\delta$ varies from $10^{-8}$.

Example 1. First we consider the BVP with Robin boundary conditions [15]:

$$
\begin{array}{r}
-\frac{\mathrm{d}^{2} u}{\mathrm{~d} x^{2}}+u=2 \cos x, \quad \pi / 2<x<\pi \\
u^{\prime}(\pi / 2)+3 u(\pi / 2)=-1, \quad u^{\prime}(\pi)+4 u(\pi)=-4,
\end{array}
$$

and the exact solution is $u(x)=\cos x$.

The BVP (11) over [0,1] is equivalently to the BVP

$$
\begin{gathered}
-\frac{1}{(\pi / 2)^{2}} \frac{\mathrm{d}^{2} u}{\mathrm{~d} x^{2}}+u=2 \cos \left(\frac{\pi}{2} x+\frac{\pi}{2}\right), 0<x<1 \\
\frac{2}{\pi} u^{\prime}(0)+3 u(0)=-1, \quad \frac{2}{\pi} u^{\prime}(1)+4 u(1)=-4
\end{gathered}
$$

Using the formula derived in equations (8) and using different number of Bernoulli polynomials, the approximate solutions are summarized in Table 1. It is observed that the accuracy is found nearly the order $10^{-6}, 10^{-9}$ and $10^{-11}$ on using 5, 7 and 9 Bernoulli polynomials, respectively.

Example 2. We consider the BVP with Dirichlet boundary conditions [1]:

$$
\begin{array}{ll}
\frac{\mathrm{d}^{2} u}{\mathrm{~d} x^{2}}+u=x^{2} \mathrm{e}^{-x}, & 0<x<10 \\
u(0)=0, & u(10)=0
\end{array}
$$

The exact solution is:

$$
\begin{aligned}
& \frac{1}{2} \mathrm{e}^{-x} \\
& {\left[1+2 x+x^{2}-\mathrm{e}^{x} \cos x-2 \mathrm{e}^{x}\left\{-\frac{\cot 10}{2}+\frac{121 \cos \mathrm{e} 10}{2 \mathrm{e}^{10}}\right\} \sin x\right]}
\end{aligned}
$$

The BVP (13) is equivalent to the BVP

$$
\frac{1}{100} \frac{\mathrm{d}^{2} u}{\mathrm{~d} x^{2}}+u=100 x^{2} \mathrm{e}^{-10 x}, \quad 0<x<1
$$

$$
u(0)=u(1)=0
$$

Using the formula derived in Equations (9), the approximate solutions, shown in Table 2 , are obtained using 8, 10 and 15 Bernoulli polynomials with accuracy up to 3, 4 and 6 significant digits, respectively. It is observed that using 21 Bernstein polynomials, the accuracy is found nearly the order of $10^{-5}$ in [1].

Example 3. In this case we consider the BVP with Dirichlet boundary conditions [4]:

$$
\begin{array}{ll}
\frac{\mathrm{d}^{2} u}{\mathrm{~d} x^{2}}=\frac{2}{x^{2}} u-\frac{1}{x}, & 2<x<3 \\
u(2)=0, & u(3)=0
\end{array}
$$

The exact solution is:

$$
u(x)=\frac{1}{38}\left[-5 x^{2}+19 x-\frac{36}{x}\right]
$$

The BVP (15) is equivalent to the BVP

$$
\begin{gathered}
\frac{\mathrm{d}^{2} u}{\mathrm{~d} x^{2}}=\frac{2}{(x+2)^{2}} u-\frac{1}{x+2}, \quad 0<x<1, \\
u(0)=0, \quad u(1)=0
\end{gathered}
$$

Using the formula derived in Equations (9), the approximate solutions, shown in Table $\mathbf{3}$, are obtained on using 5, 7 and 10 Bernoulli polynomials, and the accuracy is observed nearly 7, 8 and 9 decimal places, respectively. On the contrary, the error is obtained nearly $10^{-10}$ by Arshad [10] with $h=1 / 32$, where $h=(b-a) / N, a$ and $b$ are the endpoints of the domain and $N$ is the number of subdivision of intervals $[a, b]$.

Example 4. We consider the BVP with Neumann boundary conditions [2]:

$$
\begin{array}{r}
\frac{\mathrm{d}^{2} u}{\mathrm{~d} x^{2}}+u=-1, \quad 0 \leq x \leq 1 \\
u^{\prime}(0)=\frac{1-\cos 1}{\sin 1}, \quad u^{\prime}(1)=-\frac{1-\cos 1}{\sin 1}
\end{array}
$$

whose exact solution is, $u(x)=\cos x+\frac{1-\cos 1}{\sin 1} \sin x-1$.

Using the formula given in Equations (10), the approximate solutions, shown in Table 4, are obtained on using 5, 7 and 10 Bernoulli polynomials with the remarkable accuracy nearly the order of $10^{-7}, 10^{-10}$ and $10^{-14}$. On the other hand, Ramadan et al. [6] has found nearly the accuracy of order $10^{-6}$ and $10^{-6}$, and $10^{-8}$ on using quadratic and cubic polynomial splines, and nonpolynomial spline, respectively with $h=1 / 128$, where $h=(b-a) / N, a$ and $b$ are the endpoints of the domain and $N$ is the number of subdivision of intervals $[a, b]$. 
Table 1. Approximate solutions and absolute differences for the example 1.

\begin{tabular}{|c|c|c|c|c|c|c|}
\hline$x$ & Approximate & Absolute Error & Approximate & Absolute Error & Approximate & Absolute Error \\
\hline & \multicolumn{2}{|c|}{ Bernoulli polynomials, 5} & \multicolumn{2}{|c|}{ Bernoulli polynomials, 7} & \multicolumn{2}{|c|}{ Bernoulli polynomials, 9} \\
\hline$\pi / 2$ & 00.0000000000 & 0.0000000000 & 00.000000000 & 0.0000000000 & 0.0000000000 & 0.00000000000 \\
\hline $11 \pi / 20$ & -0.1564317160 & $2.749065 \times 10^{-6}$ & -0.1564344475 & $1.755782 \times 10^{-8}$ & -0.1564344650 & $4.925213 \times 10^{-11}$ \\
\hline $3 \pi / 5$ & -0.3090255130 & $8.518602 \times 10^{-6}$ & -0.3090169914 & $3.001562 \times 10^{-9}$ & -0.3090169944 & $5.283479 \times 10^{-11}$ \\
\hline $13 \pi / 20$ & -0.4539971315 & $6.631794 \times 10^{-6}$ & -0.4539905271 & $2.730026 \times 10^{-8}$ & -0.4539904998 & $3.642830 \times 10^{-11}$ \\
\hline $7 \pi / 10$ & -0.5877810347 & $4.217626 \times 10^{-6}$ & -0.587785252 & $7.19809 \times 10^{-10}$ & -0.5877852522 & $1.148404 \times 10^{-10}$ \\
\hline $3 \pi / 4$ & -0.7070961552 & 0.0000110000 & -0.7071067522 & $2.896640 \times 10^{-8}$ & -0.7071067812 & $9.037660 \times 10^{-12}$ \\
\hline $4 \pi / 5$ & -0.8090116456 & $5.348787 \times 10^{-6}$ & -0.8090169912 & $3.186240 \times 10^{-9}$ & -0.8090169945 & $1.525206 \times 10^{-10}$ \\
\hline $17 \pi / 20$ & -0.8910126268 & $6.102617 \times 10^{-6}$ & -0.8910065523 & $2.810947 \times 10^{-8}$ & -0.8910065241 & $4.453860 \times 10^{-11}$ \\
\hline $9 \pi / 10$ & -0.9510659385 & $9.422186 \times 10^{-6}$ & -0.9510565150 & $1.320862 \times 10^{-9}$ & -0.9510565162 & $1.337538 \times 10^{-10}$ \\
\hline$\pi$ & -1.0000000000 & 0.0000000000 & -1.0000000000 & 0.0000000000 & -1.0000000000 & 0.00000000000 \\
\hline
\end{tabular}

Table 2. Approximate solutions and absolute differences for the example 2.

\begin{tabular}{|c|c|c|c|c|c|c|}
\hline$x$ & Approximate & Absolute Error & Approximate & Absolute Error & Approximate & Absolute Error \\
\hline & \multicolumn{2}{|c|}{ Bernoulli polynomials, 8} & \multicolumn{2}{|c|}{ Bernoulli polynomials, 10} & \multicolumn{2}{|c|}{ Bernoulli polynomials, 15} \\
\hline 0 . & 0.0000000000 & 0.0000000000 & 0.0000000000 & 0.0000000000 & 0.0000000000 & 0.0000000000 \\
\hline 1. & 1.1136094978 & 0.0051685418 & 1.1187686211 & $9.418441 \times 10^{-6}$ & 1.1187829850 & $4.945487 \times 10^{-6}$ \\
\hline 2. & 1.5330008942 & 0.0100998520 & 1.5229276754 & $2.663320 \times 10^{-5}$ & 1.5228972291 & $3.813112 \times 10^{-6}$ \\
\hline 4. & -0.0413011761 & 0.0096199100 & -0.0317862308 & $1.049646 \times 10^{-4}$ & -0.0316869594 & $5.693175 \times 10^{-6}$ \\
\hline 5. & -0.7601267725 & 0.0047616938 & -0.7647484696 & $1.399968 \times 10^{-4}$ & -0.7648818892 & $6.577111 \times 10^{-6}$ \\
\hline 6. & -0.6303887189 & 0.0058561235 & -0.6363262078 & $8.136546 \times 10^{-5}$ & -0.6362508546 & $6.012205 \times 10^{-6}$ \\
\hline 7. & 0.1575008515 & 0.0046972725 & 0.1621779732 & $2.015082 \times 10^{-5}$ & 0.1622028015 & $4.677455 \times 10^{-6}$ \\
\hline 8. & 0.8534716130 & 0.0008286515 & 0.8543763192 & $7.605468 \times 10^{-5}$ & 0.8542961563 & $4.108267 \times 10^{-6}$ \\
\hline 10. & 0.0000000000 & 0.0000000000 & 0.0000000000 & 0.0000000000 & 0.0000000000 & 0.0000000000 \\
\hline
\end{tabular}

Table 3. Approximate solutions and absolute differences for the example 3 .

\begin{tabular}{|c|c|c|c|c|c|c|}
\hline$x$ & Approximate & Absolute Error & Approximate & Absolute Error & Approximate & Absolute Error \\
\hline & \multicolumn{2}{|c|}{ Bernoulli polynomials $=5$; } & \multicolumn{2}{|c|}{ Bernoulli polynomials $=7$; } & \multicolumn{2}{|c|}{ Bernoulli polynomials $=\mathbf{1 0}$; } \\
\hline 2.0 & 0.0000000000 & 0.0000000000 & 0.0000000000 & 0.0000000000 & 0.0000000000 & 0.00000000005 .349 \\
\hline 2.1 & 0.0186087702 & $2.523743 \times 10^{-7}$ & 0.0186090317 & $9.107887 \times 10^{-9}$ & 0.0186090279 & $871 \times 10^{-9}$ \\
\hline 2.2 & 0.0325370415 & $1.156379 \times 10^{-6}$ & 0.0325358850 & $1.414425 \times 10^{-10}$ & 0.0325358805 & $4.662072 \times 10^{-9}$ \\
\hline 2.3 & 0.0420487288 & $6.738722 \times 10^{-7}$ & 0.0420480426 & $1.229932 \times 10^{-8}$ & 0.0420480538 & $1.138989 \times 10^{-9}$ \\
\hline 2.4 & 0.0473677309 & $6.901458 \times 10^{-7}$ & 0.0473684233 & $2.207156 \times 10^{-9}$ & 0.0473684265 & $5.421880 \times 10^{-9}$ \\
\hline 2.5 & 0.0486829640 & $1.246501 \times 10^{-6}$ & 0.0486842230 & $1.246639 \times 10^{-8}$ & 0.0486842096 & $9.479982 \times 10^{-10}$ \\
\hline 2.6 & 0.0461533943 & $4.518647 \times 10^{-7}$ & 0.0461538458 & $3.633628 \times 10^{-10}$ & 0.0461538418 & $4.318545 \times 10^{-9}$ \\
\hline 2.7 & 0.0399130707 & $7.900046 \times 10^{-7}$ & 0.0399122691 & $1.157579 \times 10^{-8}$ & 0.0399122828 & $2.126246 \times 10^{-9}$ \\
\hline 2.8 & 0.0300761580 & $9.700405 \times 10^{-7}$ & 0.0300751896 & $1.649355 \times 10^{-9}$ & 0.0300751900 & $2.000938 \times 10^{-9}$ \\
\hline 2.9 & 0.0167419695 & $3.172309 \times 10^{-7}$ & 0.0167422939 & $7.169105 \times 10^{-9}$ & 0.0167422842 & $2.571030 \times 10^{-9}$ \\
\hline 3.0 & 0.0000000000 & 0.0000000000 & 0.0000000000 & 0.0000000000 & 0.0000000000 & 0.0000000000 \\
\hline
\end{tabular}


Table 4. Approximate solutions and absolute differences for the example 4.

\begin{tabular}{|c|c|c|c|c|c|c|}
\hline$x$ & Approximate & Absolute Error & Approximate & Absolute Error & Approximate & Absolute Error \\
\hline & \multicolumn{2}{|c|}{ Bernoulli polynomials $=5$} & \multicolumn{2}{|c|}{ Bernoulli polynomial $=7$} & \multicolumn{2}{|c|}{ Bernoulli polynomials $=\mathbf{1 0}$} \\
\hline 0.0 & 0.0000000000 & $1.153937 \times 10^{-10}$ & 0.0000000000 & 0.0000000000 & 0.0000000000 & 0.0000000000 \\
\hline 0.1 & 0.0495431439 & $2.654280 \times 10^{-7}$ & 0.0495434086 & $8.177960 \times 10^{-10}$ & 0.0495434094 & $1.932482 \times 10^{-14}$ \\
\hline 0.2 & 0.0886011150 & $9.870426 \times 10^{-7}$ & 0.0886001278 & $8.626597 \times 10^{-11}$ & 0.0886001279 & $2.672862 \times 10^{-14}$ \\
\hline 0.3 & 0.1167806021 & $6.883117 \times 10^{-7}$ & 0.1167799150 & $1.222063 \times 10^{-9}$ & 0.1167799138 & $1.992850 \times 10^{-14}$ \\
\hline 0.4 & 0.1338006690 & $5.349698 \times 10^{-7}$ & 0.1338012039 & $9.031828 \times 10^{-11}$ & 0.1338012040 & $1.013079 \times 10^{-14}$ \\
\hline 0.5 & 0.1394927538 & $1.173569 \times 10^{-6}$ & 0.1394939260 & $1.280755 \times 10^{-9}$ & 0.1394939273 & $2.942091 \times 10^{-14}$ \\
\hline 0.6 & 0.1338006690 & $5.349698 \times 10^{-7}$ & 0.1338012039 & $9.031831 \times 10^{-11}$ & 0.1338012040 & $1.010303 \times 10^{-14}$ \\
\hline 0.7 & 0.1167806021 & $6.883117 \times 10^{-7}$ & 0.1167799150 & $1.222063 \times 10^{-9}$ & 0.1167799138 & $1.981748 \times 10^{-14}$ \\
\hline 0.8 & 0.0886011150 & $9.870426 \times 10^{-7}$ & 0.0886001278 & $8.626594 \times 10^{-11}$ & 0.0886001279 & $2.670086 \times 10^{-14}$ \\
\hline 0.9 & 0.0495431439 & $2.654280 \times 10^{-7}$ & 0.0495434085 & $8.177957 \times 10^{-10}$ & 0.0495434094 & $1.942890 \times 10^{-14}$ \\
\hline 1.0 & 0.0000000000 & 0.0000000000 & 0.0000000000 & 0.0000000000 & 0.0000000000 & 0.0000000000 \\
\hline
\end{tabular}

We now also implement the procedure described in section 3 to find the numerical solutions of two nonlinear second order boundary value problems.

Example 5. We consider a nonlinear BVP with Dirichlet boundary conditions [16]

$$
\begin{aligned}
\frac{\mathrm{d}^{2} u}{\mathrm{~d} x^{2}}+\frac{1}{8} u \frac{\mathrm{d} u}{\mathrm{~d} x} & =\left(4+\frac{1}{4} x^{3}\right), 1<x<3 \\
u(1) & =17 \text { and } u(3)=43 / 3
\end{aligned}
$$

The exact solution of the problem is given by

$$
u(x)=x^{2}+\frac{16}{x}
$$

To use Bernoulli polynomials, first we convert the BVP (18) to an equivalent BVP on $[0,1]$ by replacing $x$ by $2 x+1$ such that

$$
\begin{gathered}
\frac{\mathrm{d}^{2} u}{\mathrm{~d} x^{2}}+\frac{1}{4} u \frac{\mathrm{d} u}{\mathrm{~d} x}=16+(2 x+1)^{3}, 0<x<1, \\
u(0)=17 \text { and } u(1)=43 / 3 .
\end{gathered}
$$

Assume that the approximate solution of (19) using Bernoulli polynomials is given by

$$
\tilde{u}(x)=\theta_{0}(x)+\sum_{i=2}^{n} a_{i} B r_{i}(x), \quad n \geq 2,
$$

where $\theta_{0}(x)=17-8 x / 3$ is specified by the Dirichlet boundary conditions at $x=0$ and $x=1$, and $B r_{i}(0)=B r_{i}(1)=0$ for each $i=2,3, \cdots, n$.

The weighted residual equations of (19a) corresponding to the approximation (20), given by

$$
\begin{gathered}
\int_{0}^{1}\left(\frac{\mathrm{d}^{2} \tilde{u}}{\mathrm{~d} x^{2}}+\frac{1}{4} \tilde{u} \frac{\mathrm{d} \tilde{u}}{\mathrm{~d} x}-\left[16+(2 x+1)^{3}\right]\right) B r_{k}(x) \mathrm{d} x=0 \\
k=2,3, \cdots, n .
\end{gathered}
$$

Exploiting integration by parts with minor simplifications, we obtain

$$
\begin{aligned}
& \sum_{i=2}^{n}\left[\int _ { 0 } ^ { 1 } \left[-\frac{\mathrm{d} B r_{i}}{\mathrm{~d} x} \frac{\mathrm{d} B r_{k}}{\mathrm{~d} x}+\frac{1}{4}\left(\theta_{0} \frac{\mathrm{d} B r_{i}}{\mathrm{~d} x} B r_{k}+\frac{\mathrm{d} \theta_{0}}{\mathrm{~d} x} B r_{i} B r_{k}\right)\right.\right. \\
& \left.\left.+\frac{1}{4} \sum_{j=2}^{n} a_{j}\left(B r_{i} \frac{\mathrm{d} B r_{j}}{\mathrm{~d} x} B r_{k}\right)\right] \mathrm{d} x\right] a_{i} \\
& =\int_{0}^{1}\left[\left(16+(2 x+1)^{3}\right) B r_{k}+\frac{\mathrm{d} \theta_{0}}{\mathrm{~d} x} \frac{\mathrm{d} B r_{k}}{\mathrm{~d} x}-\frac{1}{4} \theta_{0} \frac{\mathrm{d} \theta_{0}}{\mathrm{~d} x} B r_{k}\right] \mathrm{d} x \\
& k=2,3, \cdots, n
\end{aligned}
$$

The above Equations (22) are equivalent to the matrix form

$$
(D+C) A=G
$$

where the elements of the matrix $A, C, D$ and $G$ are $a_{i}, c_{i, k}, d_{i, k}$ and $g_{k}$, respectively, given by

$$
\mathrm{d}_{i, k}=\int_{0}^{1}\left[-\frac{\mathrm{d} B r_{i}}{\mathrm{~d} x} \frac{\mathrm{d} B r_{k}}{\mathrm{~d} x}+\frac{1}{4}\left(\theta_{0} \frac{\mathrm{d} B r_{i}}{\mathrm{~d} x} B r_{k}+\frac{\mathrm{d} \theta_{0}}{\mathrm{~d} x} B r_{i} B r_{k}\right)\right] \mathrm{d} x
$$

$$
c_{i, k}=\frac{1}{4} \sum_{j=2}^{n} a_{j} \int_{0}^{1}\left(B r_{i} \frac{\mathrm{d} B r_{j}}{\mathrm{~d} x} B r_{k}\right) \mathrm{d} x
$$

$g_{k}=\int_{0}^{1}\left[\left(16+(2 x+1)^{3}\right) B r_{k}+\frac{\mathrm{d} \theta_{0}}{\mathrm{~d} x} \frac{\mathrm{d} B r_{k}}{\mathrm{~d} x}-\frac{1}{4} \theta_{0} \frac{\mathrm{d} \theta_{0}}{\mathrm{~d} x} B r_{k}\right] \mathrm{d} x$

The initial values of these coefficients $a_{i}$ are obtained by applying the Galerkin method to the BVP neglecting the nonlinear term in $(19 a)$. That is, to find initial coefficients, we will solve the system

$$
D A=G
$$


where the matrices are constructed from

$$
\begin{aligned}
& \mathrm{d}_{i, k}=-\int_{0}^{1} \frac{\mathrm{d} B r_{i}}{\mathrm{~d} x} \frac{\mathrm{d} B r_{k}}{\mathrm{~d} x} \mathrm{~d} x \text { and } \\
& g_{k}=\int_{0}^{1}\left[\left(16+(2 x+1)^{3}\right) B r_{k}+\frac{\mathrm{d} \theta_{0}}{\mathrm{~d} x} \frac{\mathrm{d} B r_{k}}{\mathrm{~d} x}\right] \mathrm{d} x
\end{aligned}
$$

Once the initial values of the parameters $a_{i}$ are obtained from Equation (25a), they are substituted into Equation (23) to obtain new estimates for the values of $a_{i}$. This iteration process continues until the converged values of the unknowns are obtained. Substituting the final values of the coefficients in Equation (20), we obtain an approximate solution of the BVP (19), and if we replace $x$ by $(x-1) / 2$ in this solution we will obtain the approximate solution of the given BVP (18).

Using first 10 and 15 Bernoulli polynomials with 8 iterations, the absolute differences between exact and the approximate solutions are sown in Table 5. It is observed that the accuracy is found of the order nearly $10^{-6}$ and $10^{-8}$ on using 10 and 15 Bernoulli polynomials, respectively.

Example 6. Consider a nonlinear differential equation [9] with the Robin boundary conditions [17]:

$$
\begin{array}{r}
\frac{\mathrm{d}^{2} u}{\mathrm{~d} x^{2}}=\frac{1}{2}(1+x+u)^{3}, \quad 0<x<1 . \\
u^{\prime}(0)-u(0)=-1 / 2 \text { and } u^{\prime}(1)+u(1)=1 .
\end{array}
$$

The exact solution of the problem is given by

$$
u(x)=\frac{2}{2-x}-x-1 \text {. }
$$

In this case, solving the nonlinear BVP (26) by Modified Galerkin method, the approximate solution is assumed by

$$
u(x)=\sum_{i=0}^{n} a_{i} B r_{i}(x), \quad n \geq 1,
$$

Now following the procedures described as in exam-

\begin{tabular}{|c|c|c|c|c|}
\hline$x$ & Approximate & Absolute Error & Approximate & Absolute Error \\
\hline & \multicolumn{2}{|c|}{ Bernoulli polynomials $=10$} & \multicolumn{2}{|c|}{ Bernoulli polynomials $=15$} \\
\hline 1.0 & 17.0000000000 & 0.0000000000 & 17.0000000000 & 0.0000000000 \\
\hline 1.1 & 15.7554441298 & $1.041563 \times 10^{-5}$ & 15.7554545265 & $1.894190 \times 10^{-8}$ \\
\hline 1.2 & 14.7733332492 & $8.415722 \times 10^{-8}$ & 14.7733333734 & $4.008605 \times 10^{-8}$ \\
\hline 1.3 & 13.9977064922 & $1.418450 \times 10^{-5}$ & 13.9976922315 & $7.623683 \times 10^{-8}$ \\
\hline 1.4 & 13.3885732769 & $1.848369 \times 10^{-6}$ & 13.3885714535 & $2.496207 \times 10^{-8}$ \\
\hline 1.5 & 12.9166531985 & $1.346816 \times 10^{-5}$ & 12.9166667280 & $6.132234 \times 10^{-8}$ \\
\hline 1.6 & 12.5599891326 & $1.086740 \times 10^{-5}$ & 12.5599999558 & $4.420697 \times 10^{-8}$ \\
\hline 1.7 & 12.3017691184 & $4.412558 \times 10^{-6}$ & 12.3017646447 & $6.122859 \times 10^{-8}$ \\
\hline 1.8 & 12.1289033378 & $1.444889 \times 10^{-5}$ & 12.1288889220 & $3.310400 \times 10^{-8}$ \\
\hline 1.9 & 12.0310620046 & $9.373023 \times 10^{-6}$ & 12.0310526888 & $5.724404 \times 10^{-8}$ \\
\hline 2.0 & 11.9999952268 & $4.773188 \times 10^{-6}$ & 11.9999999739 & $2.605950 \times 10^{-8}$ \\
\hline 2.1 & 12.0290338956 & $1.372349 \times 10^{-5}$ & 12.0290475563 & $6.270952 \times 10^{-8}$ \\
\hline 2.2 & 12.1127183984 & $8.874283 \times 10^{-6}$ & 12.1127272862 & $1.346861 \times 10^{-8}$ \\
\hline 2.3 & 12.2465264382 & $4.699113 \times 10^{-6}$ & 12.2465218029 & $6.380614 \times 10^{-8}$ \\
\hline 2.4 & 12.4266794677 & $1.280107 \times 10^{-5}$ & 12.4266666577 & $9.009668 \times 10^{-9}$ \\
\hline 2.5 & 12.6500062226 & $6.222611 \times 10^{-6}$ & 12.6499999354 & $6.458229 \times 10^{-8}$ \\
\hline 2.6 & 12.9138385465 & $7.607374 \times 10^{-6}$ & 12.9138461739 & $2.007236 \times 10^{-8}$ \\
\hline 2.7 & 13.2159161538 & $9.772167 \times 10^{-6}$ & 13.2159259793 & $5.335987 \times 10^{-8}$ \\
\hline 2.8 & 13.5542901664 & $4.452108 \times 10^{-6}$ & 13.5542856601 & $5.415501 \times 10^{-8}$ \\
\hline 2.9 & 13.9272471869 & $5.807598 \times 10^{-6}$ & 13.9272414119 & $3.262486 \times 10^{-8}$ \\
\hline 3.0 & 14.3333333333 & 0.0000000000 & 14.3333333333 & 0.0000000000 \\
\hline
\end{tabular}
ple-5 and with minor simplifications, the Equation (22) leads us

$$
\begin{aligned}
& \sum_{i=0}^{n}\left[\int _ { 0 } ^ { 1 } \left[-\frac{\mathrm{d} B r_{i}}{\mathrm{~d} x} \frac{\mathrm{d} B r_{k}}{\mathrm{~d} x}-\frac{3}{2}(1+x)^{2} B r_{i} B r_{k}\right.\right. \\
& -\frac{3}{2} \sum_{j=0}^{n} a_{j}\left\{(1+x) B r_{i} B r_{j} B r_{k}\right\} \\
& \left.-\frac{1}{2} \sum_{j=0}^{n} a_{j}\left(\sum_{l-2}^{n} a_{l}\left(B r_{i} B r_{j} B r_{l} B r_{k}\right)\right)\right] \mathrm{d} x \\
& \left.-\left\{B r_{i}(1) B r_{k}(1)+B r_{i}(0) B r_{k}(0)\right\}\right] a_{i} \\
& =\frac{1}{2} \int_{0}^{1}(1+x)^{3} B r_{k} d x-\frac{1}{2} B r_{k}(0)-B r_{k}(1) \\
& k=0,1,2, \cdots, n \text {. }
\end{aligned}
$$

Table 5. Approximate solutions of example 5 using 8 iterations. 
Table 6. Approximate solutions of examples using 8 iterations.

\begin{tabular}{ccccc}
\hline $\boldsymbol{x}$ & Approximate & Absolute Error & Approximate & Absolute Error \\
\hline & \multicolumn{2}{c}{ Bernoulli polynomials, 8} & Bernoulli polynomials, 10 \\
\hline 0.0 & 00.0000000000 & 0.0000000000 & 00.0000000000 & 0.0000000000 \\
0.1 & -0.0473680463 & $3.747086 \times 10^{-7}$ & -0.0473684172 & $3.803934 \times 10^{-9}$ \\
0.2 & -0.0888891811 & $2.922364 \times 10^{-7}$ & -0.0888888959 & $6.992957 \times 10^{-9}$ \\
0.3 & -0.1235296394 & $2.276838 \times 10^{-7}$ & -0.1235293980 & $1.372571 \times 10^{-8}$ \\
0.4 & -0.1499995043 & $4.957444 \times 10^{-7}$ & -0.1500000116 & $1.157174 \times 10^{-8}$ \\
0.5 & -0.1666665743 & $9.237249 \times 10^{-8}$ & -0.1666666693 & $2.681433 \times 10^{-9}$ \\
0.6 & -0.1714291237 & $5.522990 \times 10^{-7}$ & -0.1714285563 & $1.508438 \times 10^{-8}$ \\
0.7 & -0.1615383671 & $9.448797 \times 10^{-8}$ & -0.1615384751 & $1.360781 \times 10^{-8}$ \\
0.8 & -0.1333328804 & $4.528913 \times 10^{-7}$ & -0.1333333287 & $4.621790 \times 10^{-9}$ \\
0.9 & -0.0818186682 & $4.863450 \times 10^{-7}$ & -0.0818181840 & $2.134837 \times 10^{-9}$ \\
1.0 & 00.0000000000 & 0.0000000000 & 00.0000000000 & 0.0000000000 \\
\hline
\end{tabular}

which can be written in a matrix form, similar to the system (23),

$$
(D+C+B) A=G
$$

where the elements of $A, B, C, D$ and $G$ are $a_{i}, b_{i, k}, c_{i, k}, d_{i, k}$ and $g_{k}$, respectively, given by

$$
\begin{array}{r}
\mathrm{d}_{i, k}=\int_{0}^{1}\left(\frac{\mathrm{d} B r_{i}}{\mathrm{~d} x} \frac{\mathrm{d} B r_{k}}{\mathrm{~d} x}+\frac{3}{2}(1+x)^{2} B r_{i} B r_{K}\right) \mathrm{d} x \\
+B r_{i}(1) B r_{k}(1)+B r_{i}(0) B r_{k}(0) \\
c_{i, k}=\frac{3}{2} \sum_{j=0}^{n} a_{j} \int_{0}^{1}\left\{(1+x) B r_{i} B r_{j} B r_{k}\right\} \mathrm{d} x \\
b_{i, k}=\frac{1}{2} \sum_{j=0}^{n} a_{j}\left[\sum_{l-0}^{n} a_{l} \int_{0}^{1}\left(B r_{i} B r_{j} B r_{l} B r_{k}\right) \mathrm{d} x\right] \\
g_{k}=-\frac{1}{2} \int_{0}^{1}(1+x)^{3} B r_{k} \mathrm{~d} x+\frac{1}{2} B r_{k}(0)+B r_{k}(1)
\end{array}
$$

To find the initial coefficients, on neglecting the nonlinear terms as we have done in example 5, we solve the reduced system

$$
D A=G,
$$

where the elements of $D, A$ and $G$, respectively, are now

$$
\begin{aligned}
\mathrm{d}_{i, k}= & \int_{0}^{1}\left(\frac{\mathrm{d} B r_{i}}{\mathrm{~d} x} \frac{\mathrm{d} B r_{k}}{\mathrm{~d} x}+\frac{3}{2}(1+x)^{2} B r_{i} B r_{K}\right) \mathrm{d} x \\
& +B r_{i}(1) B r_{k}(1)+B r_{i}(0) B r_{k}(0) \\
g_{k}= & -\frac{1}{2} \int_{0}^{1}(1+x)^{3} B r_{k} d x+\frac{1}{2} B r_{k}(0)+B r_{k}(1)
\end{aligned}
$$

The results are summarized in the Table 6 that obtained on using 8 and 10 Bernoulli polynomials with 8 iterations at various points of the domain of the problems. It is observed that the approximate results converge monotonically to the exact solutions.

\section{Conclusions}

We have discussed, in details, the formulation of one dimensional linear and nonlinear second order boundary value problems by Galerkin weighted residual method, using Bernoulli polynomials which have been used as the trial functions in the approximation. Some numerical examples are tested. All the mathematical formulations and numerical computations have been evaluated by MATHEMATICA code. The computed solutions are compared with the exact solutions, and we have found a good agreement with the exact solution.

\section{References}

[1] M. I. Bhatti and P. Bracken, "Solutions of Differential Equations in a Bernstein Polynomial Basis," Journal of Computational and Applied Mathematics, Vol. 205, No. 1, 2007, pp. 272-280. doi:10.1016/j.cam.2006.05.002.

[2] M. A. Ramadan, I. F. Lashien and W. K. Zahra, "Polynomial and Nonpolynomial Spline Approaches to the Numerical Solution of Second Order Boundary Value Problem," Applied Mathematics and Computation, Vol. 184, No. 2, 2007, pp. 476-484. doi:10.1016/j.amc.2006.06.053.

[3] R.A. Usmani and M. Sakai, "A Connection between Quartic Spline and Numerov Solution of a Boundary Value Problem," International Journal of Computer Mathematics, Vol. 26, No. 3, 1989, pp. 263-273. doi:10.1080/00207168908803700

[4] Arshad Khan, "Parametric Cubic Spline Solution of Two Point Boundary Value Problems," Applied Mathematics and Computation, Vol. 154, No. 1, 2004, pp. 175-182. doi:10.1016/S0096-3003(03)00701-X. 
[5] E. A. Al-Said, "Cubic Spline Method for Solving Two Point Boundary Value Problems," Korean Journal of Computational and Applied Mathematics, Vol. 5, 1998, pp. 759-770.

[6] E. A. Al-Said, "Quadratic Spline Solution of Two Point Boundary Value Problems," Journal of Natural Geometry, Vol. 12, 1997, pp. 125-134.

[7] D. J. Fyfe, "The Use of Cubic Splines in the Solution of Two Point Boundary Value Problems," The Computer Journal, Vol. 12, No. 2, 1969, pp. 188-192. doi:10.1093/comjnl/12.2.188

[8] A. K. Khalifa and J. C. Eilbeck, "Collocation with Quadratic and Cubic Splines," The IMA Journal of Numerical Analysis, Vol. 2, No. 1, 1982, pp. 111-121. doi:10.1093/imanum/2.1.111

[9] G. Mullenheim, "Solving Two-Point Boundary Value Problems with Spline Functions," The IMA Journal of Numerical Analysis, Vol. 12, No. 4, 1992, pp. 503-518. doi:10.1093/imanum/12.4.503

[10] J. Reinkenhof, "Differentiation and Integration Using Bernstein's Polynomials," International Journal for $\mathrm{Nu}$ merical Methods in Engineering, Vol. 11, No. 10, 1977, pp. 1627-1630. doi:10.1002/nme.1620111012
[11] E. Kreyszig, "Bernstein Polynomials and Numerical Integration," International Journal for Numerical Methods in Engineering, Vol. 14, No. 2, 1979, pp. 292-295. doi:10.1002/nme.1620140213

[12] R. A. Usmani, "Bounds for the Solution of a Second Order Differential Equation with Mixed Boundary Conditions," Journal of Engineering Mathematics, Vol. 9, No. 2, 1975, pp. 159-164. doi:10.1007/BF01535397

[13] B. Bialecki, "Sinc-Collocation Methods for Two Point Boundary Value Problems," The IMA Journal of $\mathrm{Nu}$ merical Analysis, Vol. 11, No. 3, 1991, pp. 357-375. doi:10.1093/imanum/11.3.357

[14] K. E. Atkinson, "An Introduction to Numerical Analysis," 2nd Edition, John Wiley and Sons, New York, 1989, pp- 284.

[15] P. E. Lewis and J. P. Ward, "The Finite Element Method, Principles and Applications," Addison-Wesley, Boston 1991.

[16] R. L. Burden and J. D. Faires, "Numerical Analysis," Brooks/Cole Publishing Co., Pacific Grove, 1992.

[17] M. K. Jain, "Numerical Solution of Differential Equations," 2nd Edition, New Age International, New Delhi, 2000 . 